\title{
On the Cotangent sums related to Estermann Zeta function and Arithmetic properties of their Arguments
}

\author{
MOULOUD GOUBI \\ Algebra and Number Theory Laboratory USTHB Algiers \\ Department of Mathematics \\ UMMTO University \\ 15000 Tizi-Ouzou \\ ALGERIA
}

Abstract: In this work we are interested by cotangent sum related to Estermann zeta function in rational arguments. In the first place we look at the maximum and the moment as they did H. Maier and M. Th. Rassias in short interval and get some interesting new identities. Afterwards, based on some recent results, we study special cases where we provide the series expansion of this sum. We end the work by the associated arithmetical problem; which consists tofindtherelationbetweenanintegeranditsinverseincongruencetheory.

Key-Words: Cotangent sums; Estermanns zeta function; Riemann zeta function; Riemann Hypothesis.

Received: November 3, 2019. Revised: March 4, 2020. Accepted: March 16, 2020. Published: March 31, 2020.

\section{Introduction}

Dedekind sums and its generalizations appear in various areas of Mathematics such as quantum theory, analytic and algebraic number theory, and topology. In the literature; several authors ([4], [5], [6] [11], [12], [14]) have already studied the cotangent sum

$$
c_{0}\left(\frac{r}{b}\right)=-\sum_{m=1}^{b-1} \frac{m}{b} \cot \left(\frac{\pi m r}{b}\right)
$$

which is related to the values at $s=\alpha=0$ of Estermann zeta function $E\left(s, \frac{r}{b}, \alpha\right)$, defined by the Dirichlet series

$$
E\left(s, \frac{r}{b}, \alpha\right)=\sum_{n \geq 1} \frac{\sigma_{\alpha}(n) \exp (2 \pi i n r / b)}{n^{s}},
$$

where $\Re(s) \geq \Re(\alpha)+1, b \geq 1,(r, b)=1$ and $\sigma_{\alpha}(n)=\sum_{d \mid n} d^{\alpha}$. Thereafter

$$
E\left(0, \frac{r}{b}, 0\right)=-\frac{i}{2} c_{0}\left(\frac{r}{b}\right)+\frac{1}{4} .
$$

By the way these sums are connected to the study of Riemann hypothesis through its relation with Vasyunin sums; defined as follows:

$$
V\left(\frac{r}{b}\right)=\sum_{m=1}^{b-1}\left\{\frac{m r}{b}\right\} \cot \left(\frac{\pi m}{b}\right),
$$

with $\{u\}=u-\lfloor u\rfloor$ for $u \in R$ set of real numbers.
Let $1 \leq \bar{r} \leq b-1$ the inverse modulo $b$ of $r$ which satisfies the relation $r \bar{r} \equiv 1(b)$. So we have

$$
c_{0}\left(\frac{r}{b}\right)=-V\left(\frac{\bar{r}}{b}\right) .
$$

$V\left(\frac{r}{b}\right)$ appears in Vasyunin formula used by V. I. Vasyunin [16] for computing the special inner product:

$$
\begin{aligned}
\left\langle e_{b}, e_{r}\right\rangle & =\frac{\log 2 \pi-\gamma}{2}\left(\frac{1}{b}+\frac{1}{r}\right)+\frac{b-r}{2 r b} \log \frac{r}{b} \\
& -\frac{\pi}{2 r b}\left(V\left(\frac{r}{b}\right)+V\left(\frac{b}{r}\right)\right)
\end{aligned}
$$

where the function $e_{b}$ is defined by

$$
e_{b}(t)=\left\{\frac{t}{b}\right\} .
$$

$\langle$,$\rangle operates on the standard Hilbert space H=$ $L^{2}\left(\left[0,+\infty\left[; t^{-2} d t\right)\right.\right.$ (see [9]), and is defined for every pair $(f, g) \in H^{2}$ by

$$
\langle f, g\rangle=\int_{0}^{+\infty} f(t) g(t) t^{-2} d t .
$$

The symmetric sum $V\left(\frac{r}{b}\right)+V\left(\frac{b}{r}\right)$ still curious, some investigations are given in the works ([6], [8]). For example we have the identity

$$
V\left(\frac{b}{r}\right)+V\left(\frac{r}{b}\right)=
$$

$\frac{1}{\pi}\left(G(b, b)+G(r, r)-2 G(b, r)+(r-b) \log \frac{b}{r}\right)$ 
where

$$
G(b, r)=\sum_{k \geq 1} \frac{b r}{k(k+1)}\left\{\frac{k}{b}\right\}\left\{\frac{k}{r}\right\} .
$$

According to Beaz-Duarte criterion ([2],[9]); Riemann hypothesis is true if and only if

$$
\lim _{n \rightarrow \infty} d_{n}=0
$$

where $\chi$ is the indicator function of the interval $\left[1,+\infty\left[\right.\right.$ and $d_{n}=\operatorname{dist}\left(\chi,\left\langle e_{1}, e_{2}, \cdots e_{n}\right\rangle\right)$ is the distance of $\chi$ to the subspace spanned by the vectors $e_{1}, e_{2}, \cdots, e_{n}$. One important result in Hilbert geometric is that

$$
d_{n}=\frac{G r\left(\chi, e_{1}, \cdots, e_{n}\right)}{G r\left(e_{1}, \cdots, e_{n}\right)}
$$

The detailed proof can be found in the work [9]. Only in [2] Baez-Duarte and al. provide that

$$
V\left(\frac{r}{b}\right)=-\frac{2}{\pi} \sum_{m=1}^{b-1} \bar{B}_{1}\left(\frac{m r}{b}\right) \psi\left(\frac{m}{b}\right)
$$

where $\psi$ is the digamma function defined in means of the series

$$
\psi(z)=-\gamma-\frac{1}{z}+\sum_{k \geq 1}\left(\frac{1}{k}-\frac{1}{k+z}\right),
$$

and $\bar{B}_{1}$ the first reduced Bernoulli polynomial given by

$$
\bar{B}_{1}(x)= \begin{cases}\{x\}-1, & \text { if } x \in R \backslash Z, \\ 0, & \text { if } x \in Z .\end{cases}
$$

We remember that $\bar{B}_{1}$ satisfies the identities

$$
\bar{B}_{1}(1-x)=-\bar{B}_{1}(x)
$$

and

$$
\sum_{r=0}^{b-1} \bar{B}_{1}\left(x+\frac{r}{b}\right)=\bar{B}_{1}(b x),
$$

which are special cases of general formulae (see [3]);

$$
B_{n}(1-x)=(-1)^{n} B_{n}(x)
$$

and

$$
\sum_{r=0}^{b-1} B_{n}\left(x+\frac{r}{b}\right)=b^{1-n} B_{n}(b x), n \in N \backslash\{0\},
$$

where $B_{n}(x)$ are Bernoulli polynomials defined in means of generating function

$$
\frac{t e^{x t}}{e^{t}-1}=\sum_{n \geq 0} \frac{B_{n}(x)}{n} t^{n},
$$

and $\bar{B}_{1}(x)=B_{1}(\{x\})$ by definition. According to identity (2) we get new reformulation of $c_{0}\left(\frac{r}{b}\right)$ given by

$$
c_{0}\left(\frac{r}{b}\right)=\frac{2}{\pi} \sum_{m=1}^{b-1} \bar{B}_{1}\left(\frac{m \bar{r}}{b}\right) \psi\left(\frac{m}{b}\right) .
$$

Notice that $\overline{b-r}=b-\bar{r}$, furthermore

$$
c_{0}\left(\frac{b-r}{b}\right)=-c_{0}\left(\frac{r}{b}\right) .
$$

The related moment

$$
\frac{1}{\phi(b)} \sum_{\substack{r, b)=1 \\ A_{0} b<r<A_{1} b}} c_{0}\left(\frac{r}{b}\right)^{2 k}, 0<A_{0}<A_{1}<1
$$

is studied in the work [13], the authors proved that

$$
\frac{1}{\phi(b)} \sum_{\substack{(r, b)=1 \\ A_{0} b<r<A_{1} b}} c_{0}\left(\frac{r}{b}\right)^{2 k}=b^{2 k} H_{k}(1+o(1)), b \rightarrow \infty,
$$

where

$$
H_{k}:=\int_{0}^{1}\left(\frac{g(x)}{\pi}\right)^{2 k} d x
$$

and

$$
g(x)=\sum_{n \geq 1} \frac{1-2\{n x\}}{n} .
$$

While looking at the work of baez-Duarte and al.[2], we deduce that the function $g$ can be written in the form

$$
g(x)=-2 \sum_{n \geq 1} \frac{\bar{B}_{1}(n x)}{n},
$$

for which the Fourier series is

$$
g(x)=\frac{2}{\pi} \sum_{n \geq 1} \frac{d(n)}{n} \sin 2 \pi n x .
$$

At rational arguments $g$ coincide with Vasyunin cotangent sum;

$$
g\left(\frac{r}{b}\right)=-\frac{\pi}{b} V\left(\frac{r}{b}\right) .
$$

Furthermore we have already proved the identity of Lemma (2.6) of the article [13]:

$$
c_{0}\left(\frac{r}{b}\right)=2 b \pi^{-2} \sum_{n \geq 1} \frac{d(n)}{n} \sin \frac{2 \pi n \bar{r}}{b} .
$$

Recently in the work [13], Helmut Maier and Michael Th. Rassias investigate the maximum of $\left|c_{0}\left(\frac{r}{b}\right)\right|$ for the value of $r$ in short interval 
]$A_{0} b,\left(A_{0}+\Delta\right) b\left[\right.$ and proved for $0<A_{0}<1$, $0<C<\frac{1}{2}, \Delta=b^{-C}$ and $0<D<\frac{1}{2}-C$ that

$$
\max _{A_{0} b<r<\left(A_{0}+\Delta\right) b}\left|c_{0}\left(\frac{r}{b}\right)\right| \leq \frac{D}{\pi} b \log b .
$$

In this work use new techniques to get general formulae concerning cotangent sums $c_{0}$ and study arithmetic properties of their arguments.

\section{Coaching of cotangent sum $c_{0}$ and associated moments}

First we revisit the work [13] and consider $r$ in the overall interval $[1, b-1]$ to estimate the maximum of $\left|c_{0}\left(\frac{r}{b}\right)\right|$ and obtain

\section{Theorem 1}

$$
\begin{array}{r}
\max _{1 \leq r \leq b-1}\left|c_{0}\left(\frac{r}{b}\right)\right| \leq \\
\frac{b-2}{\pi}\left(\frac{\gamma}{\log b}-\frac{\gamma}{b \log b}+1\right) \log b
\end{array}
$$

An improvement of result (8) is illustrated in the following corollary.

Corollary 2 For $b \geq 3$ and every $1 \leq r \leq b-1$, we have

$$
\left|c_{0}\left(\frac{r}{b}\right)\right| \leq \frac{D^{\prime}}{\pi} b \log b
$$

where $0<D^{\prime}<1$ and exactly

$$
D^{\prime}=\frac{b-2}{b}\left(\frac{\gamma}{\log b}-\frac{\gamma}{b \log b}+1\right)
$$

This corollaire is immediate, the proof is left as a sample exercices.

Let the arithmetical function $\varphi_{b}$ defined by

$$
\varphi_{b}(m)=\sum_{(r, b)=1}\left\{\frac{m r}{b}\right\},
$$

$\varphi_{b}$ is periodic with period $b$ and vanish in the set $b N$. If $b$ a prime number; $\varphi_{b}$ coincide with Euler function in $N \backslash b N$ and we have $\varphi_{b}(m)=\frac{1}{2} \varphi(b)$. According to $\varphi_{b}$ the following theorem holds.

Theorem 3 The partial sum of $c_{0}$ satisfies the following identity

$$
\begin{aligned}
\sum_{(r, b)=1} c_{0}\left(\frac{r}{b}\right) & =\frac{2}{\pi} \sum_{m=1}^{b-1} \varphi_{b}(m) \psi\left(\frac{m}{b}\right) \\
& +\frac{1}{\pi} \varphi(b)(b \log b+\gamma b-\gamma)(.11)
\end{aligned}
$$

If $b$ a prime number then

$$
\sum_{r=1}^{b-1} c_{0}\left(\frac{r}{b}\right)=0 .
$$

The restricted moment studied by $\mathrm{H}$. Maier and $\mathrm{M}$. Th. Rassias satisfies the following universal inequality independent of parameter $r$.

\section{Proposition 4}

$$
\sum_{\substack{(r, b)=1 \\ A_{0} b<r<A_{1} b}} c_{0}\left(\frac{r}{b}\right)^{2 k} \leq \frac{A_{1}-A_{0}}{\pi^{2 k}} b^{2 k+1}(\log b)^{2 k} .
$$

\subsection{Some properties of digamma function}

Before proving the last results we recall some old and new properties of digamma function. We begin by the following integral representation (see [6]).

$$
\psi\left(z_{1}\right)-\psi\left(z_{2}\right)=\int_{0}^{+\infty} \frac{e^{-z_{2} t}-e^{-z_{1} t}}{1-e^{-t}} d t
$$

which implies that

$$
\psi\left(z_{1}\right)-\psi\left(z_{2}\right)=\int_{0}^{1} \frac{t^{z_{2}-1}-t^{z_{1}-1}}{1-t} d t
$$

and

$$
\psi\left(z_{1}\right)=-\gamma+\int_{0}^{1} \frac{1-t^{z_{1}-1}}{1-t} d t .
$$

Furthermore $\psi\left(z_{1}\right)$ is negative in the interval $\left.]-\infty, 1\right]$. If $z_{1}=n$ is a positive integer; $\psi(n+1)=-\gamma+H_{n}$ where $H_{n}=\sum_{j=1}^{n} \frac{1}{j}$ is the harmonic number of order $n$, which can be written in the form

$$
H_{n}=\int_{0}^{1} \frac{1-t^{n}}{1-t} d t
$$

Finally the reflection formula $[1, \S 6.3 .7]$ which relates $\psi$ to cotangent function is given by

$$
\psi\left(1-z_{1}\right)-\psi\left(z_{1}\right)=\pi \cot \left(\pi z_{1}\right) .
$$

Let the arithmetical function $f(m)=\psi\left(\frac{m}{b}\right)$, then for $1 \leq m \leq b ; f$ is a decreasing function with $f(b)=\psi(1)=-\gamma$. From the identity (see [15, Theorem 2.2]); $\psi(x+y)-\psi(x) \geq \psi(y)$ we conclude that $f(1)=\psi\left(\frac{1}{b}\right) \leq 0$. Which means that $f(m)$ is strictly negative for $m \in\{1,2,3, \cdots, b\}$. In a recent work [10], we have proved that the partial sum of $f$ satisfies the identity

$$
\sum_{m=1}^{b} f(m)=-\gamma b-b \log b
$$


and then

$$
\lim _{b \rightarrow+\infty} \sum_{m=1}^{b} f(m)=-\infty
$$

Here we propose another proof as follows;

$$
\sum_{m=1}^{b} \psi\left(\frac{m}{b}\right)=-\gamma b+\int_{0}^{1} \frac{b-\sum_{m=1}^{b} t^{\frac{m}{b}-1}}{1-t} d t
$$

then

$$
\sum_{m=1}^{b} \psi\left(\frac{m}{b}\right)=-\gamma b+\int_{0}^{1} \frac{b-1+t^{-1}-b t^{-\frac{1}{b}}}{\left(1-t^{-\frac{1}{b}}\right)(1-t)} d t
$$

Taking a new variable $x=t^{1 / b}$, we obtain

$$
\sum_{m=1}^{b} \psi\left(\frac{m}{b}\right)=-\gamma b+b \int_{0}^{1} \frac{b\left(x^{b}-x^{b-1}\right)+1-x^{b}}{(x-1)\left(1-x^{b}\right)} d x .
$$

Finally

$$
\begin{aligned}
\sum_{m=1}^{b} \psi\left(\frac{m}{b}\right) & =-\gamma b+\int_{0}^{1}\left(\frac{b x^{b-1}}{1-x^{b}}+\frac{1}{x-1}\right) d x \\
& =-\gamma b-b \log b .
\end{aligned}
$$

Evaluating the sum

$$
\sum_{m=1}^{b} m^{p} \psi\left(\frac{m}{b}\right)
$$

for any positive integer $p$ still an open problem. In what follows an attempt of calculations for $p=1$ by using the integral representation. Remark that

$$
t\left(\frac{1-t^{b}}{1-t}\right)^{\prime}=t\left(\sum_{m=0}^{b-1} t^{m}\right)^{\prime}=\sum_{m=1}^{b-1} m t^{m}
$$

and regarding the integral representation (14) we conclude that

$$
\begin{aligned}
\sum_{m=1}^{b} m \psi\left(\frac{m}{b}\right) & =-\frac{1}{2} \gamma b(b-1) \\
& +b \int_{0}^{1} \frac{P(x)}{\left(1-x^{b}\right)(1-x)^{2}} d x
\end{aligned}
$$

where

$P(x)=\frac{1}{2} b(b-1)(1-x)^{2} x^{b-1}+b x^{b-1}(1-x)+x^{b}-1$

Furthermore

$$
\sum_{m=1}^{b} m \psi\left(\frac{m}{b}\right)=-\frac{1}{2} \gamma b(b-1)+b I(b)
$$

with

$$
I(b)=\int_{0}^{1} h(t) d x
$$

where

$h(t)=\frac{\frac{1}{2} b(b-1) x^{b-1}}{1-x^{b}}+\frac{b x^{b-1}}{\left(1-x^{b}\right)(1-x)}-\frac{1}{(1-x)^{2}}$.

Let the quantity

$$
I_{\epsilon}=\int_{0}^{\epsilon} \frac{b x^{b-1}}{\left(1-x^{b}\right)(1-x)} d x
$$

then

$$
\sum_{m=1}^{b-1} m \psi\left(\frac{m}{b}\right)=-\frac{1}{2} \gamma b(b-1)+b \lim _{\epsilon \rightarrow 1} G(\epsilon)
$$

where

$$
G(\epsilon)=I_{\epsilon}-\frac{1}{2}(b-1) \log \left(1-\epsilon^{b}\right)-\frac{1}{(1-\epsilon)}+1 .
$$

By virtue of formula (7); $c_{0}\left(\frac{1}{b}\right)$ depends on this sum and we have

$c_{0}\left(\frac{1}{b}\right)=\frac{2}{\pi b} \sum_{m=1}^{b-1} m \psi\left(\frac{m}{b}\right)+\frac{1}{\pi}(-\gamma+\gamma b+b \log b)$.

\subsection{Proof of Theorem 1, Theorem 3 and Proposition 4}

First one remarks that the Bernoulli polynomial

$$
\bar{B}_{1}(t)=\{t\}-\frac{1}{2}, t \neq 0
$$

satisfies for $1 \leq r \leq b-1$ and $b \geq 2$ the double inequality

$$
\frac{2-b}{2 b} \leq \bar{B}_{1}\left(\frac{m r}{b}\right) \leq \frac{b-2}{2 b}
$$

This result is to fact that

$$
\frac{1}{b} \leq\left\{\frac{m r}{b}\right\} \leq \frac{b-1}{b}
$$

Since $\psi\left(\frac{m}{b}\right)$ is a negative number for $1 \leq m \leq b-1$, and according to formula (4) of cotangent sum $V\left(\frac{r}{b}\right)$ we conclude that

$$
-\frac{2-b}{\pi b} \sum_{m=1}^{b-1} \psi\left(\frac{m}{b}\right) \leq V\left(\frac{r}{b}\right) \leq-\frac{b-2}{\pi b} \sum_{m=1}^{b-1} \psi\left(\frac{m}{b}\right)
$$


but we have

$$
\sum_{m=1}^{b-1} \psi\left(\frac{m}{b}\right)=\gamma-\gamma b-b \log b .
$$

Then

$\left|V\left(\frac{r}{b}\right)\right| \leq \frac{1}{\pi}\left(1-\frac{2}{b}\right)\left(\frac{\gamma}{\log b}-\frac{\gamma}{b \log b}+1\right) b \log b$.

The last inequality is independent of $r$, then we can replace $r$ by $\bar{r}$ and get the desired result (9) Theorem 1. This result is valid for every value of $b$ not forcedly large. As example for $b=2$ we deduce the well-known result $c_{0}\left(\frac{1}{2}\right)=0$.

The function $h(t)=\frac{1}{\pi}\left(1-\frac{2}{t}\right)\left(\frac{\gamma}{\log t}-\frac{\gamma}{t \log t}+1\right)$ is well defined in the interval $[2,+\infty[$ and satisfies the inequality $0 \leq h(t) \leq 1$.

In means of identity (2) the obvious symmetric identity relating $c_{0}$ to $V$ is

$\sum_{(r, b)=1} c_{0}\left(\frac{r}{b}\right)=-\sum_{(r, b)=1} V\left(\frac{\bar{r}}{b}\right)=-\sum_{(r, b)=1} V\left(\frac{r}{b}\right)$.

thus

$\sum_{(r, b)=1} c_{0}\left(\frac{r}{b}\right)=\frac{2}{\pi} \sum_{m=1}^{b-1}\left(\sum_{(r, b)=1} \bar{B}_{1}\left(\frac{m r}{b}\right)\right) \psi\left(\frac{m}{b}\right)$.

But we known that

$$
\sum_{(r, b)=1} \bar{B}_{1}\left(\frac{m r}{b}\right)=\varphi_{b}(m)-\frac{1}{2} \varphi(b)
$$

Furthermore

$$
\begin{aligned}
\sum_{(r, b)=1} c_{0}\left(\frac{r}{b}\right) & =\frac{2}{\pi} \sum_{m=1}^{b-1} \varphi_{b}(m) \psi\left(\frac{m}{b}\right) \\
& -\frac{1}{\pi} \varphi(b) \sum_{m=1}^{b-1} \psi\left(\frac{m}{b}\right),
\end{aligned}
$$

and the result (11) Theorem (3) follows.

If $b$ prime then $(m, b)=1$ for $1 \leq m \leq b-1$ and

$$
\varphi_{b}(m)=\sum_{(r, b)=1}\left\{\frac{r m}{b}\right\}=\sum_{r=1}^{b-1} \frac{r}{b},
$$

but it is well known that

$$
\sum_{r=1}^{b-1} r=\frac{b(b-1)}{2}
$$

, then $\varphi_{b}(m)=\frac{b-1}{2}$. Since the Euler function $\varphi$ satisfies the identity $\varphi(b)=b-1$ for $b$ prime; the result (12) is deduced.

Using identity (10) Corollary 2 we obtain

$$
c_{0}^{2 k}\left(\frac{r}{b}\right)=\left|c_{0}\left(\frac{r}{b}\right)\right|^{2 k} \leq \frac{D^{\prime 2 k}}{\pi^{2 k}} b^{2 k}(\log b)^{2 k} .
$$

Then

$$
\sum_{\substack{(r, b)=1 \\ A_{0} b<r<A_{1} b}} c_{0}\left(\frac{r}{b}\right)^{2 k} \leq \frac{\left(A_{1}-A_{0}\right) b}{\pi^{2 k}} b^{2 k}(\log b)^{2 k}
$$

and the identity (13) Proposition 4 follows.

\section{Special cases}

The quadratic congruences of the form $r^{2} \equiv 1(b)$ has at least two solutions $r=1$ and $r=b-1$. If $b$ is prime these solutions are unique, and $c_{0}\left(\frac{b-1}{b}\right)$ is the opposite of $c_{0}\left(\frac{1}{b}\right)$.

Several works are done on the sum $c_{0}\left(\frac{1}{b}\right)$. We recall here the most recent. In [6] we have computed the integral representation of $c_{0}\left(\frac{1}{b}\right)$ and found that

$c_{0}\left(\frac{1}{b}\right)=\frac{1}{\pi} \int_{0}^{1} \frac{(b-2) x^{b}-b x^{b-1}+b x-b+2}{(1-x)^{2}\left(x^{b}-1\right)} d x$

which can be rewritten in the form (see [10])

$$
c_{0}\left(\frac{1}{b}\right)=\frac{1}{\pi} \int_{0}^{1} \frac{\sum_{m=1}^{b-1}(b-m-1) m x^{m-1}}{1+x+\cdots+x^{b-1}} d x .
$$

Only in [10] a series expansion is given by

$$
\begin{aligned}
c_{0}\left(\frac{1}{b}\right) & =\frac{1}{\pi} b(b-1)(b-2) \\
& \times \sum_{k \geq 0} \frac{b_{k}}{(k+1)(k+2)(k+b)(k+b+1)} .
\end{aligned}
$$

The number sequence $b_{k}$ is generated by the function $\frac{1}{(1-x)^{2}\left(1-x^{p}\right)}$ i.e

$$
\frac{1}{(1-x)^{2}\left(1-x^{p}\right)}=\sum_{k \geq 0} b_{k} x^{k} ;|x|<1 .
$$

In unpublished work (M. Goubi. Series representation of a cotangent sum related to the Estermann zeta function; http://arxiv.org/abs/1903.00250), we have showed that $b_{k}$ satisfies the recursion relation

$$
b_{k}= \begin{cases}k+1, & \text { if } k<b, \\ b_{k-b}+k+1, & \text { if } k \geq b .\end{cases}
$$


Explicitly we have

$$
b_{k}=\left(k+1-\frac{b}{2}\left\lfloor\frac{k}{b}\right\rfloor\right)\left(\left\lfloor\frac{k}{b}\right\rfloor+1\right) .
$$

and for $k=i b+r$ with $i \geq 0$ and $0 \leq r \leq b-1$ we deduce that

$$
b_{i b+r}=\frac{1}{2}\left(b i^{2}+(b+2 r+2) i+2 r+2\right) .
$$

It's interesting to remember the proof of the explicit formula by using Cauchy product of series (see [7]) and associated arithmetical properties. Taking $|x|<$ 1 , it is well known that

$$
\frac{1}{1-x}=\sum_{k \geq 0} x^{k}
$$

and

$$
\frac{1}{1-x^{b}}=\sum_{k \geq 0} x^{b k}
$$

In the goal of customization we write

$$
\frac{1}{1-x^{b}}=\sum_{k \geq 0} c_{b}(k) x^{b k}
$$

with $c_{b}(k)=1$ if $b$ divides $k$ and zero otherwise. Using Cauchy product of generating functions we get

$$
\frac{1}{(1-x)^{2}}=\sum_{k \geq 0}(k+1) x^{k}
$$

and

$\frac{1}{(1-x)^{2}\left(1-x^{b}\right)}=\sum_{k \geq 0}\left(\sum_{j=0}^{k}(k-j+1) c_{b}(j)\right) x^{k}$.

But

$$
\left\lfloor\frac{k}{b}\right\rfloor b \leq\left(\left\lfloor\frac{k}{b}\right\rfloor+1\right) b
$$

then

$$
\sum_{j=0}^{k}(k-j+1) c_{b}(j)=\sum_{j=0}^{\left\lfloor\frac{k}{b}\right\rfloor}(k-j p+1),
$$

and after calculus we get the desired result.

For the sake of finding a good expression for $c_{0}\left(\frac{1}{b}\right)$ one continues to establish a variety of formulas which connect it to other sums and functions. Let the sequence $s_{k}$ given by

$s_{k}=2 k^{2}-\left[2\left(b_{k}-b_{k-1}\right)-2-b\right] k-b b_{k}-(b-2) b_{k-1}$.

According to this sequence; a connection between $c_{0}\left(\frac{1}{b}\right)$ and harmonic number $H_{b}$ is given in the following theorem.

\section{Theorem 5}

$c_{0}\left(\frac{1}{b}\right)=\frac{1}{\pi}\left(b H_{b}-3 b+1\right)-\frac{1}{\pi} \sum_{k \geq b} \frac{s_{k}}{k(k+1)}$

The proof consist to use the telescopic identity

$$
\begin{aligned}
\frac{b(b-1)(b-2)}{(k+1)(k+2)(k+b)(k+b+1)}= & \frac{b}{k+b}-\frac{b-2}{k+b+1} \\
& +\frac{b-2}{k+1}-\frac{b}{k+2}
\end{aligned}
$$

and the expression (16) to conclude that

$$
\begin{array}{r}
c_{0}\left(\frac{1}{b}\right)=\frac{1}{\pi} \sum_{k \geq 0} \frac{(2 k+3 b) b_{k}}{(k+b)(k+b+1)} \\
-\frac{1}{\pi} \sum_{k \geq 0} \frac{(2 k-b+4) b_{k}}{(k+1)(k+2)}
\end{array}
$$

and

$$
\begin{array}{r}
c_{0}\left(\frac{1}{b}\right)=\frac{1}{\pi} \sum_{k \geq b} \frac{(2 k+b) b_{k-b}}{k(k+1)} \\
-\frac{1}{\pi} \sum_{k \geq 1} \frac{(2 k-b+2) b_{k-1}}{k(k+1)} .
\end{array}
$$

Furthermore

$$
\begin{aligned}
c_{0}\left(\frac{1}{b}\right) & =\frac{1}{\pi} \sum_{k \geq b} \frac{(2 k+b) b_{k-b}-(2 k-b+2) b_{k-1}}{k(k+1)} \\
& -\frac{1}{\pi} \sum_{k=1}^{b-1} \frac{(2 k-b+2) b_{k-1}}{k(k+1)}
\end{aligned}
$$

but

$$
\begin{aligned}
\sum_{k=1}^{b-1} \frac{(2 k-b+2) b_{k-1}}{k(k+1)} & =\sum_{k=1}^{b-1} \frac{(2 k-b+2)}{k+1} \\
& =-b\left(H_{b}-1\right)+2(b-1) \\
& =-b H_{b}+3 b-2 .
\end{aligned}
$$

Finally

$$
c_{0}\left(\frac{1}{b}\right)=\frac{1}{\pi}\left(b H_{b}-3 b+1\right)-\frac{1}{\pi} \sum_{k \geq b} \frac{s_{k}}{k(k+1)}
$$

We recall that (see [8])

$$
G(b, r)=\sum_{r=1}^{b r}\left(\psi\left(\frac{r+1}{b r}\right)-\psi\left(\frac{r}{b r}\right)\right) .
$$

A new reformulation of $c_{0}\left(\frac{1}{b}\right)$ in means of the function $G$ is shown in the following proposition. 


\section{Proposition 6}

$$
\begin{array}{r}
c_{0}\left(\frac{1}{b}\right)=-\frac{1}{\pi}(\log b-\log 2) \\
+\frac{1}{\pi} \sum_{r=3}^{b}[G(r, r)+G(r-1, r-1)-2 G(r, r-1)]
\end{array}
$$

Applying the reciprocity law (3) to couple $(r-1, r)$ we obtain

$$
V\left(\frac{r-1}{r}\right)+V\left(\frac{1}{r-1}\right)=\frac{1}{\pi}\left(g(r, r-1)-\log \frac{r}{r-1}\right)
$$

where

$g(r, r-1)=G(r, r)+G(r-1, r-1)-2 G(r, r-1)$,

and then

$c_{0}\left(\frac{1}{r}\right)-c_{0}\left(\frac{1}{r-1}\right)=\frac{1}{\pi}\left(g(r, r-1)-\log \frac{r}{r-1}\right)$.

Since

$$
\sum_{r=3}^{b}\left(c_{0}\left(\frac{1}{r}\right)-c_{0}\left(\frac{1}{r-1}\right)\right)=c_{0}\left(\frac{1}{b}\right)
$$

then

$$
\pi c_{0}\left(\frac{1}{b}\right)=\sum_{r=3}^{b}\left(g(r, r-1)-\log \frac{r}{r-1}\right) .
$$

Since we have

$$
\sum_{r=3}^{b} \log \frac{r}{r-1}=\log b-\log 2
$$

the desired result holds.

\section{Associated congruence equation}

To get an explicit formula of $c_{0}$ at rational argument $\frac{r}{b}$ we must resolve the congruence equation $r \bar{r}=1$, with $1 \leq \bar{r} \leq b-1$. We know that $r^{\phi(b)} \equiv 1(b)$ and generally $r^{\phi(b)-1}$ is greater then $b$, then we deduce that $\bar{r}=b\left\{\frac{r^{\phi(b)-1}}{b}\right\}$. The inverse modulo $b$ depends on the fractional part function and we have'nt any explicit formula. Some relevant studies can be found in [17] and [18]. In [12] the authors introduced the sum

$$
Q\left(\frac{r}{b}\right)=\sum_{m=1}^{b-1} \cot \left(\frac{\pi m r}{b}\right)\left\lfloor\frac{m r}{b}\right\rfloor .
$$

and proved that

$$
c_{0}\left(\frac{r}{b}\right)=\frac{1}{r} c_{0}\left(\frac{1}{b}\right)-\frac{1}{r} Q\left(\frac{r}{b}\right) ;
$$

For $r=b-1$ we have

$$
Q\left(\frac{r}{b}\right)=-\sum_{m=1}^{b-1} m \cot \left(\frac{\pi m}{b}\right) .
$$

but

$c_{0}\left(\frac{b-1}{b}\right)=\frac{1}{b-1} c_{0}\left(\frac{1}{b}\right)-\frac{1}{b-1} Q\left(\frac{b-1}{b}\right) ;$

returning back to the definition 1 of $c_{0}\left(\frac{1}{b}\right)$ we have

$$
c_{0}\left(\frac{1}{b}\right)=-\sum_{m=1}^{b-1} \frac{m}{n} \cot \left(\frac{\pi m}{b}\right) .
$$

Let us denoting

$$
R\left(\frac{r}{b}\right)=\sum_{m=1}^{b-1}\left\lfloor\frac{m r}{b}\right\rfloor \psi\left(\frac{m}{b}\right) .
$$

In means of sums $Q\left(\frac{r}{b}\right)$ and $R\left(\frac{r}{b}\right)$; a relationship between $r$ and $\bar{r}$ is illustrated in the following proposition

\section{Proposition 7}

$$
\begin{aligned}
& c_{0}\left(\frac{1}{b}\right)=\left(c_{0}\left(\frac{1}{b}\right)+\frac{1}{\pi}(\gamma-\gamma b-b \log b)\right) r \bar{r} \\
& -\frac{1}{\pi}\left(2 R\left(\frac{\bar{r}}{b}\right)+\gamma-\gamma b-b \log b\right) r+Q\left(\frac{r}{b}\right) .
\end{aligned}
$$

If $\bar{r}$ is easy to find we are in front of another expression of $c_{0}\left(\frac{1}{b}\right)$ :

$$
\begin{array}{r}
(1-r \bar{r}) c_{0}\left(\frac{1}{b}\right)=\frac{1}{\pi}(\gamma-\gamma b-b \log b)(\bar{r}-1) r \\
-\frac{2 r}{\pi} R\left(\frac{\bar{r}}{b}\right)+Q\left(\frac{r}{b}\right) .
\end{array}
$$

The identity (7) conducts to

$$
\begin{array}{r}
c_{0}\left(\frac{r}{b}\right)=\bar{r} c_{0}\left(\frac{1}{b}\right)- \\
\frac{2}{\pi} \sum_{m=1}^{b-1}\left(\left\lfloor\frac{m \bar{r}}{b}\right\rfloor-\frac{\bar{r}-1}{2}\right) \psi\left(\frac{m}{b}\right) .
\end{array}
$$

and

$$
c_{0}\left(\frac{r}{b}\right)=\bar{r} c_{0}\left(\frac{1}{b}\right)-
$$$$
\frac{2}{\pi} \sum_{m=1}^{b-1}\left\lfloor\frac{m \bar{r}}{b}\right\rfloor \psi\left(\frac{m}{b}\right)+\frac{\bar{r}-1}{\pi} \sum_{m=1}^{b-1} \psi\left(\frac{m}{b}\right) \text {. }
$$ 
Then

$$
\begin{array}{r}
c_{0}\left(\frac{r}{b}\right)=\bar{r} c_{0}\left(\frac{1}{b}\right)-\frac{2}{\pi} \sum_{m=1}^{b-1}\left\lfloor\frac{m \bar{r}}{b}\right\rfloor \psi\left(\frac{m}{b}\right) \\
+\frac{\bar{r}-1}{\pi}(\gamma-\gamma b-b \log b) .
\end{array}
$$

Combining this result with the identity (18) we obtain

$$
\begin{aligned}
\frac{1}{r} Q\left(\frac{r}{b}\right)+\left(\bar{r}-\frac{1}{r}\right) c_{0}\left(\frac{1}{b}\right) & +\frac{\bar{r}-1}{\pi}(\gamma-\gamma b-b \log b) \\
& =\frac{2}{\pi} \sum_{m=1}^{b-1}\left\lfloor\frac{m \bar{r}}{b}\right\rfloor \psi\left(\frac{m}{b}\right) .
\end{aligned}
$$

and the desired result holds.

\section{Conclusion}

The results explained in the previous sections show that the cotangent sum $c_{0}\left(\frac{r}{b}\right)$ still curious. But important in Baez-Duarte approach for the Riemann hypothesis and related areas. For future research, it is interesting to found a good expression of $c_{0}\left(\frac{1}{b}\right)$ in order to improve some new results on general case.

\section{References:}

[1] M. Abramowitz, I. A. Stegun, Handbook of Mathematical Function with Formula, Graphs and Mathematical Tables tenth printing with corrections, United States Department of Commerce, New York, 1972.

[2] L. Báez Duarte M. Balazard, M. Landreau and E. Saias, Etude de l'autocorrlation multiplicative de la fonction partie fractionnaire, The Ramanujan Journal 9 2005, pp. 215-240.

[3] A. Bayad, Arithmetical Properties of Elliptic Bernoulli and Euler Numbers, International Journal of Algebra 4-8 2010, pp. 353-372.

[4] S. Bettin, J. B. Conrey, Period functions and cotangent sums,Algebra and Number Theory 71, 2013, pp. 215-242.

[5] S. Bettin, On the distribution of a cotangent sum, Int. Math. Res. Notices 21, 2015, pp. 1141911432.

[6] M. Goubi, A. Bayad and M. O. Hernane Explicit and asymptotic formulae for Vasyunincotangent sums,Publications de l'Institut Mathématique, Nouvelle série 102-116, 2017, pp. 155-174.

[7] M. Goubi,Successive Derivatives of Fibonacci Type Polynomials of Higher Order in Two Variables, Filomat 32-14, 2018, pp. 5149-5159
[8] A. Bayad, M. Goubi, Reciprocity formulae for generalized Dedekind-Vasyunin-cotangent sums, Math. Meth. Appl. Sci. 2018,pp. 1-17. https://doi.org/10.1002/mma.5414.

[9] A. Bayad, M. Goubi, Proof of the Möbius conjecture revisited, Proc. Jangjeon Math. Soc., 162, 2013, pp. 237-243.

[10] M. Goubi, Series expansion of a cotangent sum related to Estermann zeta function, Kragujevac Journal of Mathematics, 45-3, 2021, pp. 343352.

[11] H. Maier, M. Th. Rassias,Explicit estimates of sums related to the NymanBeurling criterion for the Riemann Hypothesis, Journal of Functional Analysis https://doi.org/10.1016/j.jfa.2018.06.022 (arXiv:1806.05070).

[12] H. Maier, M. Th. Rassias, Generalizations of a cotangent sum associated to the Estermann zeta function, Communications in Contemporary Mathematics 18-1, 2016.

[13] H. Maier, M. Th. Rassias, The maximun of cotangent sums related to Estermann zeta function in rational numbers in short intervals, $A p$ plicable Analysis and Discrete Mathematics 11, 2017, pp. 166-176.

[14] M. Th. Rassias, A cotangent sum related to zeros of the Estermann zeta function, Applied Mathematics and Computation 240, 2014, pp. 161167.

[15] W. T. Sulaiman, Turan inequalities for the digamma and polygamma functions, South Asian J. Math. 1-2, 2011, 49-55.

[16] V. I. Vasyunin, On a biorthogonal system associated with the Riemann hypothesis, Algebra $i$ Analiz 7-3, 1995, pp. 118-135.

[17] F. Ghanim, New Study of Classes of HurwitzZeta Function Related to Integral Operator WSEAS Transactions on Mathematics 13, 2014, pp. 477-483.

[18] P. G. Brown, The Modified Mobius Function WSEAS Transactions on Computers 1-12, 2013, pp. 1-7 